$\Longrightarrow$ Taylor \& Francis Taylor \& Francis Group http://taylorandfrancis.com 


\title{
13
}

\section{SHOWING WHAT IT LOOKS LIKE}

\author{
Teaching students to use \\ diagrams in problem solving, \\ communication, and thinking
}

\author{
Emmanuel Manalo, Yuri Uesaka, Ouhao Chen, \\ and Hiroaki Ayabe
}

\section{Summary}

Cultivating the ability to use multiple representations is considered very important in 21st-century education. Students should be able to employ not only verbal representations but also visual representations such as diagrams to enable effective organization, understanding, and communication of information. However, despite this acknowledged importance, instruction in diagram use is rarely provided to students. In this chapter, we describe methods we have developed for the provision of such instruction and refer to evidence for their effectiveness, not only in improving students' spontaneity in using diagrams, but also in promoting deeper learning, communicative competencies, and critical thinking.

\section{Introduction}

The ability to use multiple representations of concepts and tasks is considered to be one of the key competencies that people need to develop in order to operate effectively in 21st-century environments (e.g., National Research Council, 2012). Multiple representations here pertain to the use of not only words, printed or spoken (i.e., verbal representations), but also diagrams, pictures, animation, and other forms of visual representations, as well as numerical, mathematical, scientific, and other forms of recognizable symbols and notations. In this chapter, we will use the term diagrams to broadly and inclusively refer to visual or graphic representations, including drawings, illustrations, tables and other arrays, flow charts, and graphs. When lines or arrows connect three or more words - thereby creating a flow or organizational chart - such a representation would also count as a diagram. 
A number of authors have explained the efficacy of using diagrams. Perhaps one of the most well-known of these explanations is Larkin and Simon's (1987), in which they pointed out that diagrams have a computational advantage over sentential representations because they "group together all information that is used together" and thus "support a large number of perceptual inferences" (p. 98). In simple terms this means that, because diagrams group information together so that we can see connections and relationships much easier, they help us to draw inferences and to understand much more efficiently compared to when information is presented serially, like in sentences (where connections and relationships are usually not as immediately obvious because of spatial, and possibly temporal, distance).

When diagrams are used appropriately together with words in learning and communicative contexts, they can greatly enhance comprehension and learning outcomes because the combination facilitates the use of both the verbal and visual channels of working memory (e.g., Mayer, 2009; Mayer \& Moreno, 1998, 2003). When diagrams are used in problem solving contexts, they help toward generating correct solutions because they translate the terms of the problem (usually given as verbal statements) into a representation that makes sense and is easier to understand (Beitzel \& Staley, 2015; Hembree, 1992; Jitendra, Griffin, Haria, Leh, Adams, \& Kaduvettoor, 2007; Tversky \& Kessell, 2014).

Despite the generally acknowledged value of using diagrams in a wide range of learning and communicative contexts, the construction and use of diagrams is not usually taught explicitly in schools. Teachers may demonstrate the use of diagrams to students when explaining information or solving problems on the board, but they rarely go through the steps involved in diagram construction, or clarify the conditions that may apply in determining what diagrams to use and when they should be used. Much of formal education emphasizes the cultivation of verbal forms of expressing knowledge and ideas (i.e., the use of words, spoken and written), with the cultivation of the corresponding or complementary visual forms of expression largely neglected.

Perhaps not surprisingly, there are a number of problems that have been identified regarding student construction and use of diagrams. The most serious of these is lack of spontaneity in use (e.g., Dufour-Janvier, Bernarz, \& Belanger, 1987; Manalo, Uesaka, Pérez-Kriz, Kato, \& Fukaya, 2013; Uesaka \& Manalo, 2017; Uesaka, Manalo, \& Ichikawa, 2007; van Garderen, Scheuermann, \& Jackson, 2012): unless required or prompted to use them, the majority of students tend not to use diagrams even in situations where diagram use would be deemed advantageous (e.g., solving difficult math word problems, constructing explanations of hardto-imagine or complicated information for others). Because in most everyday life situations (outside of school) there are no requirements or prompts for using diagrams, this lack of spontaneity means that many students are likely to miss out on the potential benefits of using diagrams. Another serious problem is that when students do use diagrams, many fail to use the appropriate diagrams or to draw correct inferences from them (e.g., Cox, 1996; Uesaka \& Manalo, 2006; 
van Garderen et al., 2012). There are also problems relating to student use of diagrams that are provided to them: for example, Cromley, Snyder-Hogan, and Luciw-Dubas (2010) found that many students ignore or only superficially look over diagrams provided in their textbooks, suggesting that they are unlikely to fully benefit from those diagrams. However, in this chapter we will focus particularly on problems and issues relating to diagrams that students construct, rather than those provided to them.

Findings from our own research on the promotion of effective strategy use suggest that when two crucial conditions are missing, students do not manifest spontaneity in the use of such strategies. Those conditions are that they must appreciate the value of employing the strategy, and they must possess adequate knowledge and skills in using that strategy (e.g., Manalo, Uesaka, \& Chinn, 2017; Uesaka \& Manalo, 2017). In the following section, we will describe methods that teachers can use to address such deficits, and hence to promote the desired spontaneity particularly in students' use of diagrams.

\section{Descriptions of methods}

\section{Cultivating appreciation of the value of diagram use}

\section{Telling them the value}

To get students to realize that using diagrams can benefit their work, teachers can directly tell them. For example, we found it effective to provide verbal encouragement to students who had failed to correctly solve a math word problem. We simply told them that they could have been more successful in solving that problem had they used an appropriate diagram: students who received such encouragement showed higher subsequent diagram use (Uesaka, Manalo, \& Ichikawa, 2010). We also found it effective to provide a hint in the written feedback given to students for an explanation homework they completed. After receiving the hint that "including diagrams could make your explanations easier to understand," the students' rate of diagram inclusion in their subsequent homework significantly increased (Manalo \& Uesaka, 2016). We should note that this increase occurred despite the fact that the students were aware that no specific grade points were allocated for diagram use in their homework.

\section{Using interactive peer instruction}

Teachers can also cultivate appreciation of the value of diagram use indirectly by getting students to work interactively with each other in peer instruction or explanation tasks. In a study involving mathematics word problem solving, we assigned pairs of junior high school students different problems to solve, and then afterward asked them to take turns at teaching each other how they solved the problem they had been assigned (Uesaka \& Manalo, 2007). We found that many of the students not only used diagrams during the peer instruction session, but 
also in subsequent problem solving tasks that were given to them to solve individually. We found their use of diagrams to be significantly higher than students who were in a control group and received the exact same problems to solve, but not the opportunities for peer instruction. Our examination of the protocols generated during the peer instruction sessions suggested that, in such situations, verbal explanations alone often prove inadequate and students have to resort to using illustrations or other forms of visual explanations to clarify how they solved the problem. This kind of experience likely made them realize that constructing diagrams was helpful in explaining. Additionally, through being able to explain how to solve the problems more easily, the students may have come to realize the utility that diagram use brings to such problem solving.

We found similar effects in a study in which we asked students (university undergraduates this time) to take turns at explaining to each other the contents of a passage they had read (they were given different passages to read). Again, we found that when peer explanations were interactive, spontaneous diagram use was significantly higher compared to noninteractive peer explanations in which the students prepared and recorded their explanations for each other (Uesaka \& Manalo, 2014). Our examination of the interactive explanation protocols revealed that students often received feedback from their interlocutors indicating that what they had said was not sufficiently understood, which made the explainers use illustrations and/or schematic diagrams to clarify details of what they were attempting to convey.

\section{Cultivating knowledge and skills in diagram construction and use}

\section{Providing the necessary instruction}

In teaching diagram construction and use to students, teachers need to facilitate the development of three kinds of knowledge: declarative (i.e., knowing that), procedural (knowing how), and conditional (knowing when) - as Paris, Lipson, and Wixson (1983) explained regarding the requirements of strategy use. For example, students need to know that constructing a table or equivalent array could be helpful when attempting to solve a math word problem in which there is a rule-bound or pattern-based change in quantities occurring and some future quantity needs to be calculated, or that including an illustration is helpful when the student needs to communicate how something appears, or where items or entities are located in relation to each other. Thus, in teaching diagram use for problem solving, students need to know and understand that, depending on the type of problem presented, a particular kind of diagram could help in solving it (e.g., Zahner \& Corter, 2010). In a recent study that we conducted in a real classroom context (Ayabe \& Manalo, 2018), we focused on the use of three kinds of diagrams: line diagrams, tables, and graphs. We used a good part of an entire class session to teach the use of each kind, first presenting examples 
of the corresponding types of problems and clarifying their requirements, next explaining how and why that kind of diagram in particular is helpful in solving such a problem, and then providing a demonstration of the steps taken in such solving.

In another study, which we also conducted in a real classroom context (Manalo \& Uesaka, 2016), we provided instruction on the use of diagrams in written communication for other people. First, we explained the general reasons why diagrams can be helpful: we pointed out that diagramming the structure and organization of target information can clarify and confirm our understanding of it, as well as help us identify parts that we may not fully understand. We referred to research about using both verbal and visual channels of working memory when diagrams are used in combination with words (e.g., Mayer, 2009) - thus, including appropriate diagrams can make our explanations easier for other people to understand and learn. After that, we explained more specifically - and showed examples of - the kinds of diagrams that are helpful when we need to illustrate (e.g., the appearance of objects, spatial layout), to provide an overview or structure, to show process or cause-and-effect relationships, and to compare and contrast. Again, the aim was for students to know and understand that, depending on the purpose and content of the communication, the use of an appropriate kind of diagram can enhance the clarity and effectiveness of that communication (e.g., Novick \& Hurley, 2001; Tversky, 2011; Xing, Corter, \& Zahner, 2016).

\section{Providing opportunities for practice}

As noted, the development of procedural knowledge is also necessary. One intervention we have found effective for this purpose is to provide guided practice in the construction and use of each kind of diagram. Hence, in the Ayabe and Manalo (2018) study, following instruction in the use of each kind of diagram (e.g., line diagrams), the students were given practice in solving problems for which the use of that kind of diagram would be helpful. In the Manalo and Uesaka (2016) study, during the practice phase of the intervention, the students were not only given practice during class to use the kinds of diagrams they had received instruction in, they were also given homework that required them to look for information they had learned which could be explained more effectively with the use of each of those diagrams - and to construct the appropriate diagrams. The importance of practice was highlighted in the finding of this study that the student participants did not make sufficient improvements in the spontaneity of their diagram use until they had received opportunities for practice. This makes sense because even if students acquire declarative knowledge about diagrams and know that they could be helpful, they would be unlikely to use them unless they have sufficient procedural knowledge and confidence in knowing how to use them - which is exactly what the provision of practice promotes.

Cultivating procedural knowledge about when to use diagrams is also crucial, and such cultivation is usually embedded in teaching students about the kinds 
of diagrams and their applications, and in providing opportunities for practice. For instance, when teaching diagram use for math word problem solving, one important indicator that we should alert students to is when they cannot immediately conceptualize how to solve a given problem: this should serve as a signal to them that they should attempt alternative strategies such as constructing an appropriate diagram to re-represent the terms of the problem so that they would be able to gain insight about how to solve it. When instructing students about the different kinds of diagrams that are helpful in problem solving, that instruction should include explanations of when each kind may be appropriate to use. For example, constructing graphs may be helpful when two or more quantities are changing and some comparison or decision needs to be made based on those changes (e.g., Corter \& Zahner, 2007; Zahner \& Corter, 2010). Explaining how those conditions apply to the problems that students are given for practice is also crucial so that they can better appreciate and understand how those conditions appear and can be identified.

In teaching the use of diagrams for more effective communication of information to others, similar clarification of the conditions that would make particular kinds of diagrams helpful is important. For instance, in the Manalo and Uesaka (2016) study we mentioned previously, we explained to the students that diagrams depicting process or cause-and-effect relationships (e.g., flow charts) should be used when the reader of the information needs to understand how something works, the steps required in a particular procedure, or to identify/clarify the causes and/or the effects contained in the information being explained. We elaborated by explaining that such diagrams effectively address certain questions, which include: What happens? How does it work? What causes it? What is the effect?

\section{Providing opportunities for active comparison}

In an earlier study (Uesaka \& Manalo, 2006), we found that the use of active comparison is effective in helping students correctly determine the choice of diagrams to use. Active comparison here pertains to an activity that was provided following students' problem solving sessions, during which they compared several kinds of representations (in this study: formulas, tables, graphs) they had used in solving different problems (the students conducted this activity mostly on their own, using worksheets provided by the teacher). The teacher asked them to consider when each kind of representation was most appropriate to use - and $w h y$, including the merits corresponding to each. The results of this study showed that students who received this additional activity constructed more appropriate diagrams in solving problems they were later administered. In a subsequent assessment of conditional knowledge, they also provided more abstract and detailed descriptions about the uses of diagrams in problem solving (e.g., "When looking for a rule about increasing values, using a table is effective"). These findings suggest that actively comparing several examples facilitates the formation of the necessary abstract rules, beyond each of the concrete examples dealt with. 
Hence, students develop an understanding of when each kind of diagram might be effective to use (i.e., the conditional knowledge for use).

\section{Evidence for effectiveness}

In the previous section, we described methods we have designed and found effective for promoting student use of diagrams. While promotion of such use is in itself very much a worthwhile goal, an even more desirable goal is to demonstrate that such use in turn leads to beneficial learning outcomes. In this section, therefore, we describe evidence indicating that the promotion of diagram use leads to deeper learning, communicative competencies, and critical thinking.

\section{Promotion of deeper learning}

Deeper learning can be described as "the process through which an individual becomes capable of taking what was learned in one situation and applying it to new situations (i.e., transfer) ... The product of deeper learning is transferable knowledge, including content knowledge in a domain and knowledge of how, why, and when to apply this knowledge to answer questions and solve problems" (National Research Council, 2012, pp. 5-6). Our research findings indicate that through the cultivation of students' appreciation of the value of diagram use, as well as their knowledge and skills in constructing and using such diagram, this kind of transfer occurs. Students not only evidence spontaneous use of diagrams in new situations, but their use of diagrams also comprises an integral part of their successful application of content knowledge (e.g., in mathematics, in education studies) to solve new problems and answer new questions. For example, in the Ayabe and Manalo (2018) study, following the provision of instruction and practice, not only did the students use diagrams more spontaneously in subsequent new problems, their correct answer rates also increased.

This kind of transfer was likewise demonstrated in the Manalo and Uesaka (2016) study, in which the focus of the intervention provided was on the use of diagrams to enhance the quality of written explanations in homework tasks. Following the interventions of providing a hint, an instruction session, and practice in diagram use, the student participants evidenced more spontaneous use of diagrams in subsequent homework tasks. Hence, they were able to effectively incorporate diagrams in constructing written explanations of new topics they were learning. However, further to this, the majority of the students also later evidenced transfer to an explanation task administered in class (i.e., not for homework - hence, a different task setting) and in a final test at the end of the semester. They were therefore incorporating diagrams in constructing written explanations in different tasks given in the course.

In Figure 13.1, we represent the components and steps involved in this kind of transfer. In the original learning situation, the intervention provided promotes students' diagram use competence and predisposition; this helps improve their 


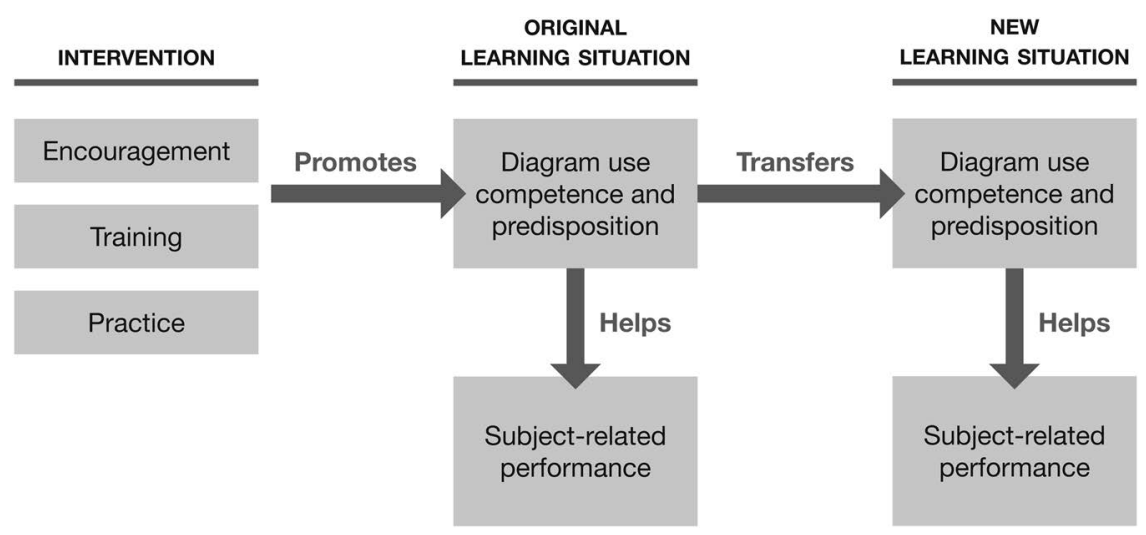

FIGURE 13.1 Transfer as evidence of deeper learning, achieved through promotion of diagram use competence and predisposition.

subject-related performance in that learning situation (e.g., they can more successfully solve math word problems, or more effectively explain information they have learned in their course). This successful application entails the integration of strategy knowledge (e.g., how to use diagrams) and content knowledge in a domain (e.g., how to solve word problems in math). Through this successful diagram application, students not only develop their knowledge about diagrams, they also get to appreciate their value. The diagram use competence and predisposition thus transfers to new learning situations; in other words, equipped with some knowledge about how to use diagrams - and presumably some appreciation of the associated benefits - the students use diagrams in new situations. As a consequence, they are able to improve their subject-related performance in those new learning situations (e.g., they can successfully solve new problems, explain different kinds of information, and complete other tasks that all have some degree of variation from what they had dealt with in the original learning situation).

\section{Promotion of communicative competence}

Our research findings indicate that with the use of diagrams, students are able to encode more of the key information in notes and explanations they construct. In one study (Chen, Manalo, \& She, 2019), we found that junior high school students who were asked to draw a diagram to represent key points from scientific information they were given to read, managed to encode more of the key points compared to students who were asked to write a summary instead. In another more recent study we (first and third authors of this chapter, teacher collaborators) have been working on with undergraduate university students as participants, preliminary results indicate that when these students $(n=45)$ were asked to include at least one diagram in written explanations they produced of what they had been given to read, they were able to encode more of the key points from the reading materials 


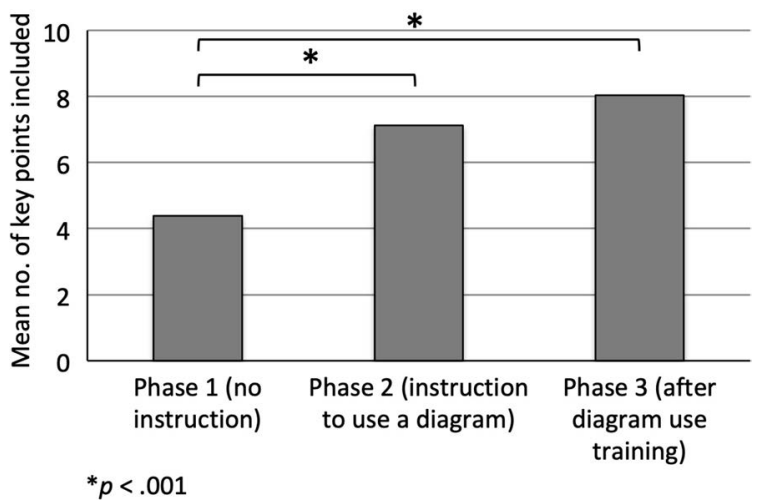

FIGURE 13.2 Mean number of key points students were able to include in written explanations they produced during the three phases of the study.

compared to baseline levels, during which they received no instruction to use a diagram. This result is shown in Figure 13.2, where phase $1=$ "no instruction to use a diagram," phase 2 = "instruction to use at least one diagram," and phase $3=$ "instruction to use at least one diagram, following training in how to use diagrams." The dependent variable is the number of key points from the reading materials the students were able to include in their written explanations (appropriate measures were taken to ensure equivalence and counterbalancing of the reading materials used). Analysis of variance revealed a significant phase effect, $F(2,42)=22.006, p<.001, \eta_{p}{ }^{2}=.51$; with the means in phases 2 and 3 being significantly higher than in phase 1 , both at $p<.001$. These results suggest that when students are asked to incorporate diagrams in writing explanations of what they have read - even without training in how to effectively use diagrams for such purposes - their capacity for encoding important information can increase.

With training in how to appropriately use diagrams in explaining information, we found increases not only in the quantity of diagrams that undergraduate university students $(n=19)$ spontaneously included in written explanations but also in the variety of diagrams they were including (Manalo, Tsuda, \& Dryer, in press). We found a significant phase effect for inclusion of each type of diagram (i.e., illustration, overview, process, and comparison diagrams) across four phases from baseline (no training) through to post-training-plus-practice [i.e., for illustration: $F(3,60)=7.19, p=.0003, \eta_{p}^{2}=.27$; for overview: $F(3,60)=$ $10.19, p=.001, \eta_{p}{ }^{2}=.34$; for process: $F(3,60)=26.96, p<.0001, \eta_{p}^{2}=.57$; for comparison: $\left.F(3,60)=4.89, p=.004, \eta_{p}^{2}=.20\right]$. For all diagram types, the mean inclusion value at the post-training-plus-practice phase was significantly higher than at baseline, all at $p<.05$. In other words, following training and practice in how to use them, the students were using more types of diagrams in explaining. This is understandable, considering that training would have provided them with more knowledge about types of diagrams and when (for what purposes) to use them. Furthermore, we found that the number of diagrams the students included 
in their explanations negatively correlated with word count $(r=-.38, p=.056)$ and verb-count $(r=-.43, p=.035)$ in those explanations (both correlations indicate at least medium-size effects). This means that the more they used diagrams, the fewer words they used and the less complicated their sentence constructions tended to be (i.e., as indicated by the verb count, which can be used as one indicator of complexity in language production, cf. Manalo \& Sheppard, 2016). However, a crucial point is that, despite the lower word and verb counts, the number of key points they included in their explanations did not decrease. This suggests that diagram use can promote efficiency in the construction of written explanations: if we include diagrams to convey what we want to communicate, we may not need to use as many words or to use structures that are as complex. This finding could have useful applications - which ought to be explored in future research - particularly in the cultivation of communicative competency in students who have some language use limitations (e.g., those who have to use a second or foreign language they are not so proficient in, those who have language-based learning disabilities such as dyslexia or dysgraphia).

\section{Promotion of critical thinking}

Where the promotion of critical thinking is concerned, research evidence shows that encouraging students to use diagrams can enable them to think more critically about information they are presented. More specifically, in Uesaka, Igarashi, and Suetsugu (2016), we found that junior high school students who received encouragement to use tables in generating and discussing arguments for and against controversial propositions (e.g., whether high school students should be permitted to hold part-time jobs) were subsequently better able to produce argumentation in which multiple perspectives about the proposition were effectively integrated, compared to students who received no such encouragement. This finding lends support to an earlier finding reported by Nussbaum (2008) that argumentation "vee diagrams" (a form of diagram that Nussbaum created) can promote argument-counterargument integration in students' reflective writing. However, our study additionally demonstrated that more ubiquitous diagrams - in this case, tables - can just as effectively serve the purpose of facilitating critical thought. Furthermore, in our study we were able to show that providing encouragement for and instruction in the use of tables for such a task can lead to significant increases in students' spontaneous decisions to use tables in a subsequent similar task (for which the students were able to freely choose whether to use a diagram or not).

We have also observed from classroom teaching situations that encouraging students to use diagrams can facilitate their ability to integrate various strands of information they are learning, and to draw inferences - both of which are important components of critical thinking (e.g., Facione, 1990). For example, in an undergraduate educational psychology course that the first author teaches, students are provided instruction on the basics of the brain structures and the 


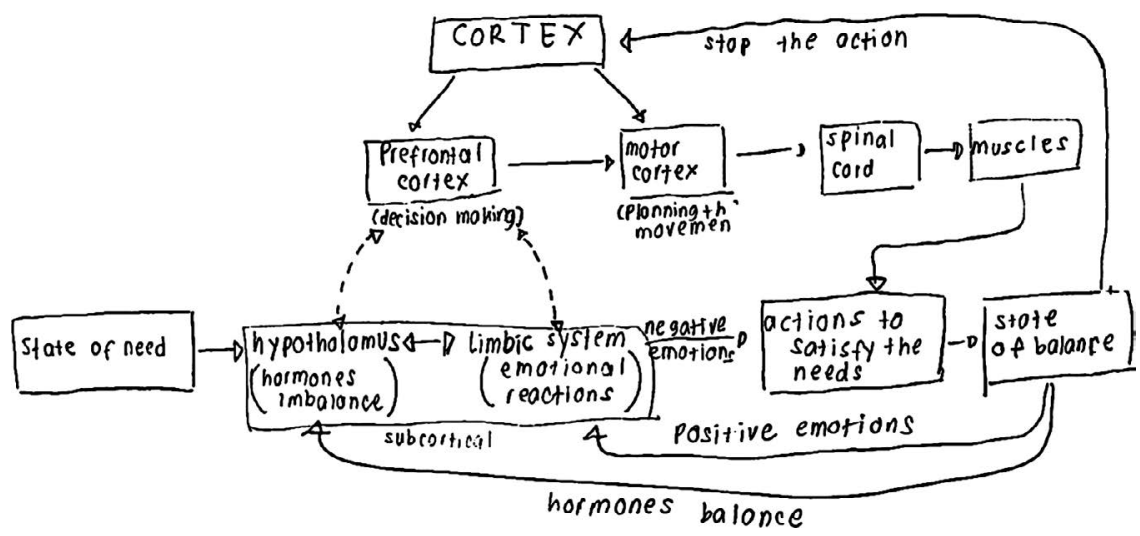

FIGURE 13.3 Example of student-generated diagram from a class exercise that required students to integrate and draw inferences from what they had learned.

functions generally attributed to different parts of the brain (e.g., the cortex and subcortical areas). They also learn about the maintenance of equilibrium and needs reduction, and how emotions/affect are also involved. But they need to make sense of how these different components are connected, so one class exercise they are given is to construct a diagram that would show the processes and connections from the emergence of "a state of need" to the satisfaction of that need in a socially acceptable way. To do this, they need to consider what happens when a human is in a state of need, and what follows after that - considering all the possibly relevant pieces of information they have learned. This is not an easy task for most students but, usually after several diagrammatic iterations, they are able to produce a diagram like the one shown in Figure 13.3. They therefore come to understand the basic mechanisms involved in this process of needs satisfaction in humans. We believe that constructing a diagram is crucial in facilitating this kind of understanding as it requires students to translate or re-represent what they know and what they have learned, as well as to clarify or make explicit what connections (e.g., arrows) might serve or mean in such representations.

Another example of a diagram produced by a student is shown in Figure 13.4. This one is from an undergraduate course in education studies, also taught by the first author. The students had received introductory lectures on comparative education and culture in the classroom and, during this particular class exercise, they were asked to consider how to connect or find relationships between the ideas those two topic areas contain. They were also asked to consider how those ideas might contribute to their own understanding of what is important in the provision of education, which is an issue discussed throughout the course. In this exercise, they were not explicitly asked to construct a diagram (many students, for example, simply jotted down bullet points), but this student spontaneously 


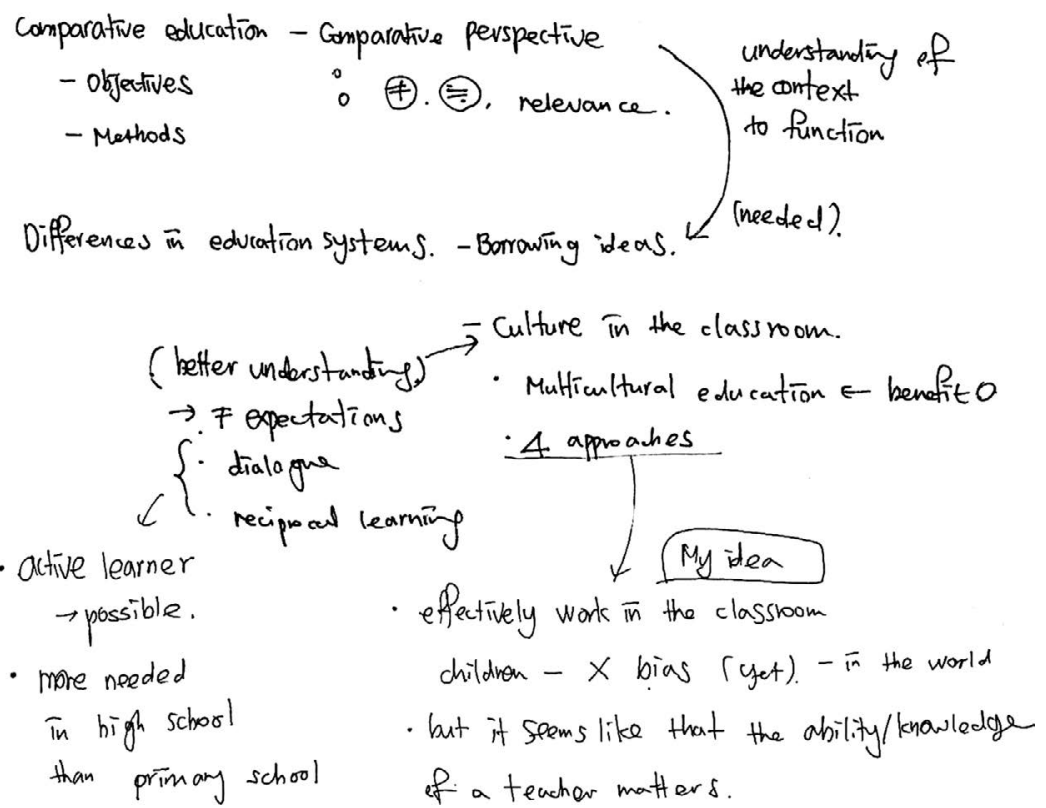

FIGURE 13.4 Another example of student-generated diagram from a class exercise that required students to consider connections between ideas and draw inferences from what they had learned.

constructed one. The diagram includes her own ideas (shown in the bottom part of the diagram): the inferences she had made about the possibility of developing more active learners (and how this may be needed more in high school), and how the introduction of culture in the classroom may be effectively undertaken because of children being - as yet - relatively unaffected by bias (but that the knowledge and ability of teachers would matter). We believe that this diagram - with the inferences drawn by the student - is another good example of how diagram use can be facilitative of critical thought. We frequently observe such facilitation when students diagrammatically represent what they have learned.

\section{Conclusion: Abstracting, linking, clarifying}

In this chapter, we have described research-based methods for promoting student use of diagrams. We have also summarized evidence indicating that these methods are effective for promoting spontaneity in diagram use, and that diagram use in turn can lead to the promotion of deeper learning, communicative competence, and critical thinking.

But why is diagram construction particularly beneficial for student learning? We believe that the answer to this question lies in diagram construction facilitating 
three mechanisms that are essential to meaningful learning: abstracting, linking, and clarifying. We mentioned earlier that in problem solving, the use of diagrams is helpful because it translates the terms of the problem into a form that is easier to understand (e.g., Beitzel \& Staley, 2015; Hembree, 1992; Jitendra et al., 2007; Tversky \& Kessell, 2014). However, to construct a useful diagram, the student needs to identify and select relevant components of the problem (and to discard irrelevant ones), and to recombine those relevant components in a way that makes sense and that would summarize the situation of the problem. In effect, therefore, the student has to abstract, or create a schematic representation (Barsalou, 2003; Tversky, 2011; Tversky \& Kessell, 2014). Abstraction is necessary for meaning apprehension and construction and, as Saitta and Zucker (2013, p. 31) pointed out, "without abstraction no high level thinking would be possible." The important point here though is that diagram construction by its very nature facilitates abstraction, which must be one crucial reason why it promotes deeper learning.

The second reason is that diagram construction facilitates linking, or the establishment of connections between different parts of the issue under consideration. Here, both the process of constructing links and the resulting product of integrated (connected) parts are important. To be able to construct links, the student needs to understand the meaning and significance of the parts (otherwise, useful connections cannot be made) - hence that process directly contributes to the apprehension of meaning. But the resulting diagram in itself, with the parts arranged and connected to make sense, is valuable in terms of showing together all the parts that are to be used together - and thus, as Larkin and Simon (1987) noted, a diagram supports a large number of perceptual inferences. The important point here is that, through such linking, diagrams not only support the apprehension of meaning, they also support drawing of inferences - which is necessary for critical thinking (e.g., Facione, 1990).

The third reason we consider important in explaining why diagram construction is beneficial to student learning is that it clarifies the information under consideration. Through the use of a diagram, a student can for example clarify for another student how to solve a problem, or how something works (e.g., Uesaka \& Manalo, 2007, 2014). But diagrams can also clarify information for oneself: as Manalo and Uesaka (2014) reported, students are more likely to spontaneously construct diagrams when making notes for their own use. This then raises the question of why diagrams clarify, and one simple answer to this - which has been mentioned earlier - is that adding a visual representation (the diagram) enables the use of the visual channel of working memory in combination with the verbal channel, where verbal information is processed (e.g., Mayer, 2009). To put this another way, instead of just hearing or reading about the information, we also get to see what it looks like - making it easier to understand. Thus, through the use of diagrams, students can improve their communicative competence: such use can enable them to more successfully convey the intended message to others, as well as to their own selves. 
However, apart from enabling the use of both channels of working memory, diagrams also clarify by making abstract meanings and ideas more concrete (e.g., Tversky, 2011; Tversky \& Kessell, 2014) - portraying how those meanings and ideas that previously lacked concrete form could look like or could be imagined. Tversky (2011) additionally pointed out that, not only do diagrams abstract and schematize by omitting what is deemed inessential, they also exaggerate and elaborate - in effect distorting the "truth" to make it clearer. Through such distortions (e.g., making important components bigger or more prominent; sharpening distinctions; adding arrows, labels, and other markers), diagrams repackage meanings and ideas for the consumption of human attention, which is known to be selective, ignoring much of incoming information (e.g., Schneider \& Shiffrin, 1977). Hence, diagrams clarify the target information because they concretize and enhance salience of what requires attention.

\section{Acknowledgement}

The authors would like to thank Christoph Daniel Schulze for the construction of the diagram shown in Figure 13.1.

\section{References}

Ayabe, H., \& Manalo, E. (2018, June). Can spontaneous diagram use be promoted in math word problem solving? Paper presented at Diagrams 2018: The 10th International Conference on the Theory and Application of Diagrams, Edinburgh, UK.

Barsalou, L. W. (2003). Abstraction in perceptual symbol systems. Philosophical Transactions of the Royal Society B, 358, 1177-1187.

Beitzel, B. D., \& Staley, R. K. (2015). The efficacy of using diagrams when solving probability word problems in college. Journal of Experimental Education, 83, 130-145.

Chen, O., Manalo, E., \& She,Y. (2019). Examining the influence of expertise on the effectiveness of diagramming and summarising when studying scientific materials. Educational Studies, 45, 57-71.

Corter, J. E., \& Zahner, D. C. (2007). Use of external visual representations in probability problem solving. Statistics Education Research Journal, 6, 22-50.

Cox, R. (1996). Analytical reasoning with multiple external representations (Unpublished doctoral thesis). University of Edinburgh, UK.

Cromley, J. G., Snyder-Hogan, L. E., \& Luciw-Dubas, U. A. (2010). Cognitive activities in complex science text and diagrams. Contemporary Educational Psychology, 35, 59-74.

Dufour-Janvier, B., Bernarz, N., \& Belanger, M. (1987). Pedagogical considerations concerning the problem of representation. In C. Janvier (Ed.), Problems of representation in the teaching and learning of mathematics (pp. 110-120). Hillsdale, NJ: Erlbaum.

Facione, P.A. (1990). Critical thinking: A statement of expert consensus for purposes of educational assessment and instruction:The Delphi Report. Berkeley, CA: Academic Press.

Hembree, R. (1992). Experiments and relational studies in problem solving: A meta-analysis. Journal for Research in Mathematics Education, 23, 242-273.

Jitendra, A. K., Griffin, C. C., Haria, P., Leh, J., Adams, A., \& Kaduvettoor, A. (2007). A comparison of single and multiple strategy instruction on third-grade students' mathematical problem solving. Journal of Educational Psychology, 99, 115-127. 
Larkin, J. H., \& Simon, H. A. (1987). Why a diagram is (sometimes) worth ten thousand words. Cognitive Science, 11, 65-99.

Manalo, E., \& Sheppard, C. (2016). How might language affect critical thinking performance? Thinking Skills and Creativity, 21, 41-49.

Manalo, E., Tsuda, A., \& Dryer, R. (in press). The effect of cultivating diagram use on the quality of EFL students' written explanations. Thinking Skills and Creativity.

Manalo, E., \& Uesaka, Y. (2014). Students' spontaneous use of diagrams in written communication: Understanding variations according to purpose and cognitive cost entailed. Lecture Notes in Artificial Intelligence, 8578, 78-92.

Manalo, E., \& Uesaka,Y. (2016). Hint, instruction, and practice:The necessary components in promoting spontaneous diagram use in students' written work? Lecture Notes in Artificial Intelligence, 9781, 157-171.

Manalo, E., Uesaka, Y., \& Chinn, C. A. (Eds.) (2017). Promoting spontaneous use of learning and reasoning strategies: Theory, research, and practice for effective transfer. London and New York: Routledge.

Manalo, E., Uesaka,Y., Pérez-Kriz, S., Kato, M., \& Fukaya, T. (2013). Science and engineering students' use of diagrams during note taking versus explanation. Educational Studies, 39, $118-123$.

Mayer, R. E. (2009). Multimedia learning (2nd ed.). New York: Cambridge University Press.

Mayer, R. E., \& Moreno, R. (1998). A split-attention effect in multimedia learning: Evidence for dual processing systems in working memory. Journal of Educational Psychology, 90, 312-320.

Mayer, R. E., \& Moreno, R. (2003). Nine ways to reduce cognitive load in multimedia learning. Educational Psychologist, 38, 43-52.

National Research Council. (2012). Education for life and work: Developing transferable knowledge and skills in the 21st century. Committee on Defining Deeper Learning and 21st Century Skills, J.W. Pellegrino and M. L. Hilton (Eds.). Washington, DC:The National Academies Press.

Novick, L. R., \& Hurley, S. M. (2001). To matrix, network, or hierarchy, that is the question. Cognitive Psychology, 42, 158-216.

Nussbaum, E. M. (2008). Using argumentation vee diagrams (AVDs) for promoting argument-counterargument integration in reflective writing. Journal of Educational Psychology, 100, 549-565.

Paris, S. G., Lipson, M.Y., \& Wixson, K. K. (1983). Becoming a strategic reader. Contemporary Education Psychology, 8, 293-316.

Saitta, L., \& Zucker,J.-D. (2013). Abstraction in artificial intelligence and complex systems. New York: Springer-Verlag.

Schneider, W., \& Shiffrin, R. M. (1977). Controlled and automatic human information processing: I. Detection, search, and attention. Psychological Review, 84, 1-66.

Tversky, B. (2011).Visualizing thought. Topics in Cognitive Science, 3, 499-535.

Tversky, B., \& Kessell, A. (2014). Thinking in action. Pragmatics \& Cognition, 22, 206-223.

Uesaka, Y., Igarashi, M., \& Suetsugu, R. (2016). Promoting multi-perspective integration as a 21st century skill: The effects of instructional methods encouraging students' spontaneous use of tables for organizing information. Lecture Notes in Artificial Intelligence, 9781, 172-186.

Uesaka, Y., \& Manalo, E. (2006). Active comparison as a means of promoting the development of abstract conditional knowledge and appropriate choice of diagrams in math word problem solving. Lecture Notes in Computer Science, 4045, 181-195.

Uesaka,Y., \& Manalo, E. (2007). Peer instruction as a way of promoting spontaneous use of diagrams when solving math word problems. In D. S. McNamara \& J. G. Trafton (Eds.), Proceedings of the 29th Annual Cognitive Science Society (pp. 677-682). Austin, TX: Cognitive Science Society. 


\section{Manalo, Uesaka, Chen, and Ayabe}

Uesaka,Y., \& Manalo, E. (2014). How communicative learning situations influence students' use of diagrams: Focusing on the spontaneous construction of diagrams and student protocols during explanation. Lecture Notes in Artificial Intelligence, 8578, 93-107.

Uesaka,Y., \& Manalo, E. (2017). How to address students' lack of spontaneity in diagram use: Eliciting educational principles for the promotion of spontaneous learning strategy use in general. In E. Manalo, Y. Uesaka, \& C. A. Chinn (Eds.), Promoting spontaneous use of learning and reasoning strategies: Theory, research, and practice for effective transfer (pp. 62-76). London and New York: Routledge.

Uesaka,Y., Manalo, E., \& Ichikawa, S. (2007). What kinds of perceptions and daily learning behaviors promote students' use of diagrams in mathematics problem solving? Learning and Instruction, 17, 322-335.

Uesaka, Y., Manalo, E., \& Ichikawa, S. (2010). The effects of perception of efficacy and diagram construction skills on students' spontaneous use of diagrams when solving math word problems. Lecture Notes in Artificial Intelligence, 6170, 197-211.

van Garderen, D., Scheuermann, A., \& Jackson, C. (2012). Examining how students with diverse abilities use diagrams to solve mathematics word problems. Learning Disability Quarterly, 36, 145-160.

Xing, C., Corter, J. E., \& Zahner, D. (2016). Diagrams affect choice of strategy in probability problem solving. Lecture Notes in Artificial Intelligence, 9781, 3-16.

Zahner, D., \& Corter, J. E. (2010).The process of probability problem solving: Use of external visual representations. Mathematical Thinking and Learning, 12, 177-204. 